\title{
Instruments optimizations for low energy Gamma-ray detection
}

\author{
Gonzalo Rodriguez ${ }^{1, a}$ and Aldo Morselli ${ }^{1}$ \\ ${ }^{1}$ INFN Roma Tor Vergata, Rome
}

\begin{abstract}
There is an experimental gap in the study of the non-thermal universe in the photon energy range from 0.3 to $100 \mathrm{MeV}$. We have analyzed the performance of a detector with unprecedented sensitivity, angular and energy resolution and combined with polarimetric capability to study of the most powerful Galactic and extragalactic sources and with a line sensitivity in the $\mathrm{MeV}$ energy range two orders of magnitude better than previous generation of instruments that can determine the origin of key isotopes fundamental for the understanding of supernova explosion and the chemical evolution of our Galaxy.
\end{abstract}

\section{Introduction}

The energy domain between 0.3 and $100 \mathrm{MeV}$ covers a crucial range of nuclear gamma-ray lines produced by radioactive decay, nuclear collision, positron annihilation, or neutron capture, which makes it as special for high-energy astronomy as optical spectroscopy is for phenomena related to atomic physics. Also some objects in the Universe have their peak emissivity at photon energies between 0.3 and $100 \mathrm{MeV}$ (e.g. gamma-ray bursts, blazars, pulsars, etc.), meaning that it is in this energy band that their physical properties can be directly studied. This energy range is also known to feature a characteristic spectral turn-over associated to hadronic emission from pion decay, that can be use to distinguishing leptonic from hadronic processes.

e-ASTROGAM [1] is a gamma-ray space mission that have been proposed as the M5 Medium-size mission of the European Space Agency. It is a gamma-ray instrument that inherits from predecessors such as AGILE [2] and Fermi [3], as well as from the MEGA [4] prototype, but it takes full advantages of recent progresses in silicon detectors and readout microelectronics to achieve measurement of the energy and 3D position of each interaction within the detectors with an excellent spectral and spatial resolution. The main innovative feature of the e-ASTROGAM mission is the capability of joint detection in the Compton $(0.3-30 \mathrm{MeV})$ and pair $(>10 \mathrm{MeV})$ energy ranges in a single integrated instrument. The mission aims at improving the sensitivity in the medium-energy gamma-ray domain by one to two orders of magnitude compared to previous missions.

\section{The e-ASTROGAM telescope}

The e-ASTROGAM telescope is made up of three detection system, see Figure 1, a silicon Tracker in which the cosmic gamma rays undergo a Compton scattering or a pair conversion, a Calorimeter

a e-mail: grferna@roma2.infn.it 
to absorb and measure the energy of the secondary particles, and an anti-coincidence (AC) system to veto the prompt-reaction background induced by charged particles. The telescope has a size of $110 \times 110 \times 80 \mathrm{~cm}^{3}$ and a mass of $820 \mathrm{~kg}$.
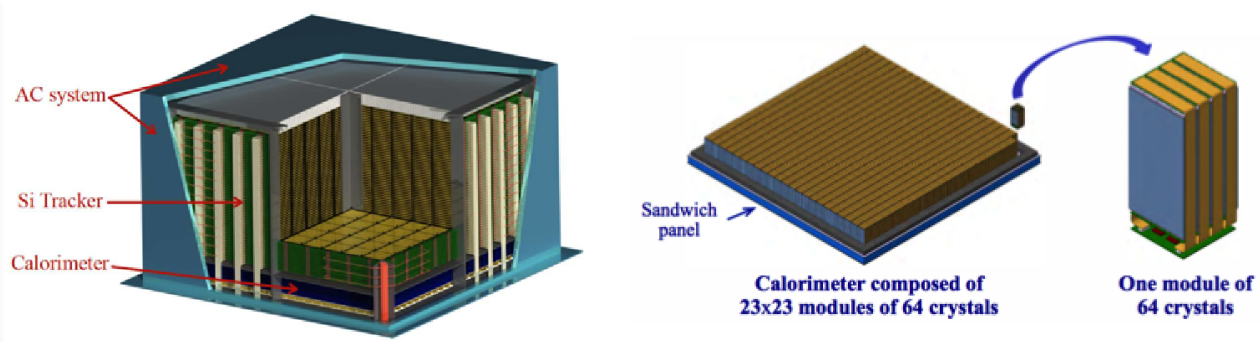

Figure 1: Overview of the e-ASTROGAM payload showing the silicon Tracker, the Calorimeter and the Anti-coincidence system.

The e-ASTROGAM Tracker is a double-sided strip detectors (DSSD) that comprises 5600 DSSDs arranged in 56 layers. It is divided in four units of 5x5 DSSDs, the detectors being wire bonded strip to strip to form 2-D ladders. The interlayer distance is $10 \mathrm{~mm}$. Each DSSD has a geometric area of $9.5 \times 9.5 \mathrm{~cm}^{2}$, a thickness of $500 \mu \mathrm{m}$, and a strip pitch of $240 \mu \mathrm{m}$. The total detection area amounts to $9025 \mathrm{~cm}^{2}$ and the total Si thickness to $2.8 \mathrm{~cm}$, which corresponds to 0.3 radiation length on axis.

The Calorimeter is a pixelated detector made of a high- $\mathrm{Z}$ scintillation material ? Thallium activated Cesium Iodide. It consists of an array of 33,856 parallelepiped bars of CsI(Tl) of $8 \mathrm{~cm}$ length and $5 \times 5 \mathrm{~mm}^{2}$ cross section, read out by silicon drift detectors (SDDs) at both ends, arranged in an array of $529(=23 \times 23)$ elementary modules comprising each 64 crystals. The Calorimeter thickness, $8 \mathrm{~cm}$ of $\mathrm{CsI}(\mathrm{Tl})$, makes it a 4.3 radiation length detector having an absorption probability of a 1-MeV photon on axis of $88 \%$.

The third main detector of the e-ASTROGAM payload consists of an Anti-coincidence (AC) system made of segmented panels of plastic scintillators covering the top and four lateral sides of the instrument, requiring a total active area of about $4.7 \mathrm{~m}^{2}$. The AC detector is segmented in 33 plastic tiles (6 tiles per lateral side and 9 tiles for the top). All scintillator tiles are coupled to silicon photomultipliers (SiPM) by optical fibers.

\section{Silicon Tracker: Geometry optimization in the Compton regime}

We have focus our optimization studies on the silicon tracker geometry. We have perform the simulations, the reconstruction and analysis of the events using the MEGAlib framework [5]. MEGAlib was develop to simulate tracker detectors in the Compton regime, and recently was update to include also the pair production regime. The detector is simulated using a wrapped of the well know Geant 4 framework [6]. The output of the simulations is reconstructed using the tools revan and mimrec.

In the energy range from 0.3 to $5 \mathrm{MeV}$ we measured the properties of the incident photon using the Compton effect. From the interaction we have two products, a photon and an electron. If the electron have enough energy will escape the silicon layer and will be detect as it hits other layers. Otherwise we will only have the hits from the photon. We have to distinguish between events with electron tracking and without electron tracking. This will have implications in the angular resolution. 


\subsection{Energy Resolution - Photopeak events}
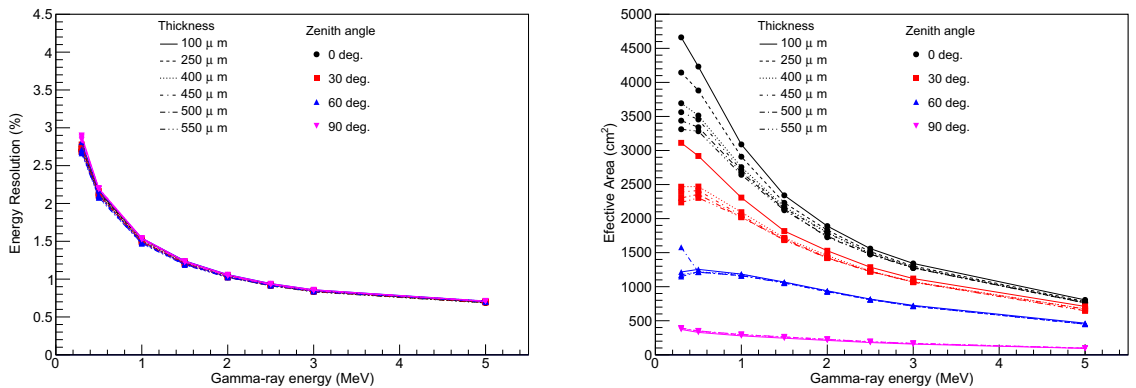

Figure 2: Energy resolution and Effective area from the photopeak area

We have to use the photopeak events to calculate the energy resolution in the Compton regime. In the Figure 2 we show the results, in the left panel we see that the energy resolution does not depends on any geometrical parameters use in the simulation. We have observe an energy resolution of $3 \%$ at low energies that decrease to about $1 \%$ at $5 \mathrm{MeV}$. In the right panel we show the dependence of the effective area as a function of the energy for different thickness and zenith angles.

\subsection{Angular Resolution - Compton events}

For the angular resolution we have to take into account the events reconstructed w/o electron tracking. For simplicity we show the results for events simulated with zenith angle at 0 degrees.
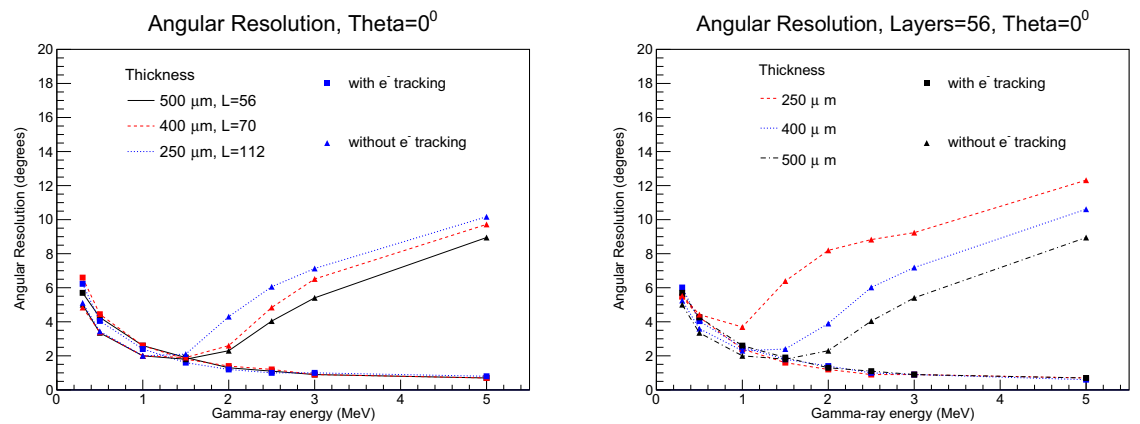

Figure 3: Angular resolution from the reconstruction of Compton events, Left panel: Fix the radiation length. Right panel: Fix the number of layers.

In the Figure 3 we show, on the left panel, the results for 3 different thickness when we have fixed the number of total radiation lengths to $0.3 \mathrm{X}_{0}$. For events without electron tracking, triangles points, we observe that at approximately $1.5 \mathrm{MeV}$ the resolutions start to get worst for all thickness. This is not the case for the reconstructed events with electron tracking, square points, the angular resolutions gets better as the energy increase, going from $6^{\circ}$ at $0.3 \mathrm{MeV}$ to $1^{\circ}$ at $5 \mathrm{MeV}$. For energies below 1.5 $\mathrm{MeV}$ both reconstruction give approximately the same angular reconstruction. 
In the right panel, we show the results for geometries with different thickness but the same number of layer, in this case 56. For events with electron tracking we observe the same results when the total radiation length was fixed. We have found a different situation when we have reconstructed events with no electron tracking. In this case depending on the thickness the angular resolutions start getting worst at different energies. We can see that for $250 \mu \mathrm{m}$ at energies less than $1 \mathrm{MeV}$ the angular resolutions start decreasing, but for 400 and $500 \mu \mathrm{m}$ this behaviour start at $1.5 \mathrm{MeV}$ or above.

It is clear that we have to make an event selection to obtain the best angular resolution as a function of the energy and for each simulated geometry.
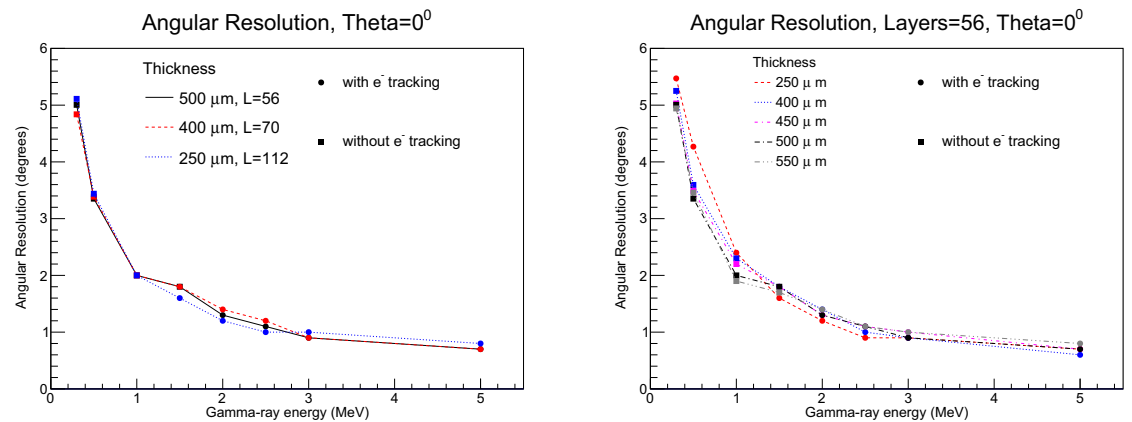

Figure 4: Angular resolution, after event selection, from the reconstruction of Compton events, Left panel: Fix the radiation length. Right panel: Fix the number of layers.

In the Figure 4 we show the results of the angular resolution after the event selection. As we can observe regardless of the geometry we have obtain the same angular resolution within the uncertainties. The angular resolution goes from $5^{\circ}$ at $0.3 \mathrm{MeV}$ to $1^{\circ}$ at $5 \mathrm{MeV}$.
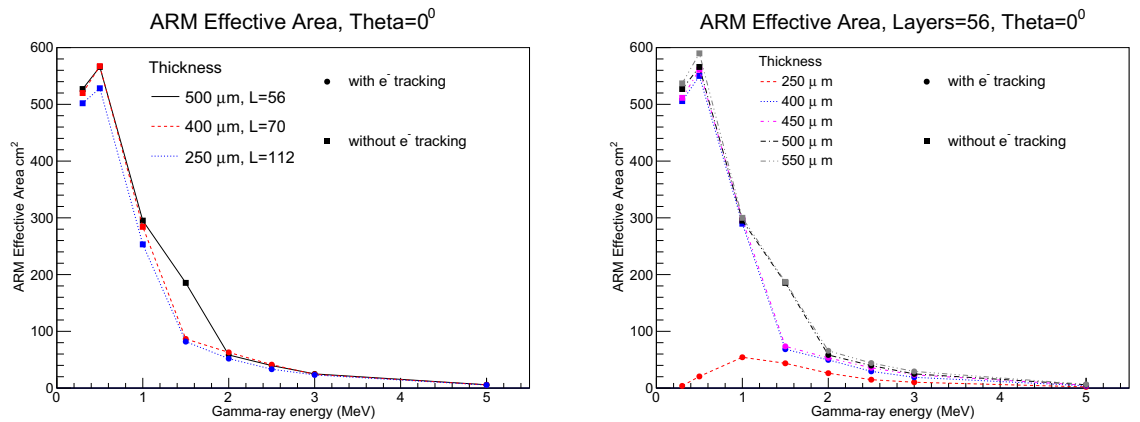

Figure 5: Effective area from the reconstruction of Compton events, Left panel: Fix the radiation length. Right panel: Fix the number of layers.

Using the event selection that minimizes the angular resolution we show in the Figure 5 the effective area. In the left panel, when the number of total radiation lengths is fixed we have obtain the same effective area independent of the simulated geometry. In the right panel, we have kept the number of layer fix to 56, in this case we observe that, for low energies, for thickness of $250 \mu \mathrm{m}$ the effective area drop dramatically in comparison with 400 and $500 \mu \mathrm{m}$. 


\section{Conclusions}

We have simulated different geometries for the eASTROGAM silicon tracker using MEGAlib framework. We have studied the Compton and Pair production regime by simulates different energies range from 0.3 to $5 \mathrm{MeV}$.

In the Compton regime we have found that the energy resolution does not depend on the simulate geometry.

For the angular resolution we have to make an event selection between reconstructed events w/o electron tracking to obtain the best possible resolution. After the selection the angular resolution is independent of the geometry. We have found difference in the effective area when the number of interactions lengths is smaller than $0.3 \mathrm{X}_{0}$, we have observed that, for low energies, for thickness of $250 \mu \mathrm{m}$ the effective area drop dramatically in comparison with 400 and $500 \mu \mathrm{m}$. The similar study in the pair production regime is on-going

\section{Acknowledgements}

The research leading to these results has received funding from the European Union's Horizon 2020 Programme under the AHEAD project (grant agreement n. 654215)

\section{References}

[1] A. De Angelis et al., Science with e-ASTROGAM (A space mission for MeV-GeV gammaray astrophysics) Journal of High Energy Astrophysics Volume 19, August 2018, Pages 1-106 https://doi.org/10.1016/j.jheap.2018.07.001 [arXiv:1711.01265]

[2] Tavani, M., Barbiellini, G., Argan, A., et al., The AGILE Mission, Astronomy and Astrophysics 502, 995-1013 (Aug. 2009).

[3] Atwood, W. B., Abdo, A. A., Ackermann, M., et al., The Large Area Telescope on the Fermi Gamma-Ray Space Telescope Mission, Astrophysical Journal 697, 1071-1102 (June 2009).

[4] Kanbach, G., Andritschke, R., Zoglauer, A., et al., Development and calibration of the tracking Compton/Pair telescope MEGA, Nuclear Instruments and Methods in Physics Research A 541, 310-322 (Apr. 2005).

[5] A.Zoglauer et al. MEGAlib - The Medium Energy Gamma-ray Astronomy Library, New Astronomy Reviews, Volume 50, Issues 7-8, October 2006

[6] S.Agostinelli et al, Geant4 - a simulation toolkit, Nuclear Instruments and Methods in Physics Research A 506 (2003) 250-303 